\title{
Population groups can hold critical clues
}

London. As geneticists try to unravel the basis of ever more complex disorders, the search for informative population groupswhether defined ethnically, geographically or by common medical history-is assuming increasing importance.

Several thousand human diseases exhibit classic mendelian (that is, recessive or dominant) inheritance, and are presumably caused by defects in single genes. But many other single-gene diseases do not follow strict mendelian patterns of inheritance. In these cases, environmental factors influence who gets sick, and the terms 'genetic predisposition' and 'susceptibility genes' are often used. Not all individuals with a particular defective gene will thus develop disease, although those who do are overwhelmingly likely to have inherited the defective gene.

Other disorders are caused by the interaction between several genes; in such 'complex disorders', the disease can be caused by a collection of genetic alterations, none of which is in itself harmful, and any one of which only slightly increases an individual's risk of disease. Here in particular, the disease does not follow simple mendelian inheritance patterns; there is no simple correlation between genotype and phenotype, and identifying the genes involved is a difficult task.

Unfortunately, most common diseases such as diabetes and asthma, as well as major killers such as cancer and heart disease, are complex disorders. But the hope that unravelling their genetic basis will lead to improved treatment or ultimately a cure has made the hunt for the genes involved a priority for researchers in academic institutions and the pharmaceutical industry.

Whatever the condition, the gene must be singled out from 80,000 others on the human genome, a feat usually performed by identifying where on the genome the gene lies. The process requires both raw material, in the form of DNA samples from related groups of individuals in whom the disease is common, and genetic markers highly variable short sequences of DNA at unique, known locations.

Such markers can be used to map disease-causing genes in many ways. All rely on the idea that if a marker lies close to the gene in question, one form of the marker is likely to be inherited along with the gene in an affected family or inbred population.

Once two markers flanking the gene have been found, they can be used as 'entry-points' for cloning DNA from the region, which in turn can be used as a source of candidate disease genes. Comparing the DNA sequence of such genes in affected and unaffected relatives leads eventually to identifying the gene whose defects are associated with the disease.

In principle, it should be easy to tell whether a gene is defective only in affected individuals. In practice, the human genome contains many polymorphisms (different forms of the same gene), and the sequence of a gene will often vary slightly from person to person unless the individuals in question share a recent common ancestor.

Most of these differences do not affect gene function. But their frequency can make it hard to identify a disease gene on the basis of sequence alterations alone.

Analysis of data from the more complex mapping techniques generally requires sophisticated computer software, and even then, very large areas of DNA must often be sequenced to identify the gene in question.

IMAGE
UNAVAILABLE
FOR COPYRIGHT
REASONS

Vital samples: blood supplies key data.

But the number of known markers, the quality of the software and the speed of sequencing have all improved substantially over the past five years. Most geneticists now consider the availability of suitable DNA samples to be the limiting factor in the study of genetic diseases.

In the past, geneticists have concentrated on single-gene diseases. Using extensive pedigrees, they have identified how the disease is inherited and compared the genotype of affected and unaffected individuals. About 500 human genes have been mapped in this way, more than 10 per cent of which have been successfully cloned.

The study of complex disorders is more difficult, as unaffected individuals may still carry the predisposing genetic alteration. Mapping studies must therefore concentrate on affected relatives, searching for genomic regions that they share more often than random inheritance would predict.

In affected families, the method most commonly used is the analysis of affected sibling pairs, known as 'sib pairs'; a recent search for genes affecting susceptibility to diabetes involved over 500 such pairs. Sib-pair analysis is now being carried out for most complex diseases.

But finding a sufficient number of sib pairs for a particular disease can be difficult, and the chromosomal region defined is seldom small enough to make identification of the gene involved easy.
Researchers are therefore turning increasingly to genetically isolated populations. Such populations often have only a small number of founding members, and a certain degree of inbreeding. Individuals therefore share significant parts of their genomes (although variation increases with time), and genetic alterations predisposing to disease tend to be inherited together with their neighbouring DNA.

As with sib-pair analysis, scanning the genomes of affected individuals for conserved chromosomal regions can be an efficient way of mapping genes of interest, sometimes down to a region small enough to allow cloning.

Nor is this the only advantage offered by genetically isolated populations. For example, variations in any one component of a complex system may be more obvious if the other (genetic and environmental) components are constant among most individuals in a group, making susceptibility genes easier to spot. A lower level of 'background' polymorphism can also help to single out such genes from among several candidates.

The Finns are a prominent example. The founders of the population arrived in Finland only about 2,000 years ago (75 generations), and the population went through a drastic decline as recently as the late seventeenth century, implying a further reduction in overall polymorphism.

Until recently, the Finns mixed little with other groups, thus limiting their genetic diversity. Furthermore, Finland has exceptional family records dating back to the seventeenth century.

Such favourable conditions have already led to the identification of genetic defects underlying several single-gene diseases in the Finnish population, and there is general optimism that this population will also be useful in the study of complex diseases such as schizophrenia and diabetes.

The advantages of such populations have encouraged scientists and pharmaceutical companies to search systematically for new remote populations with high incidences of particular disorders. One example that promises to help find asthma genes is the highly inbred population of Tristan da Cunha, a small island in the South Atlantic, one third of whose roughly 300 inhabitants suffer from the disease.

Not every disease will have a 'perfect' island. But countries such as India and China, with remote regions where population groups have little geographical mobility and a high degree of inbreeding, may well prove to be genetic goldmines. The ethical and economic problems posed by exploiting such resources will therefore be an inescapable feature of genetic research for some time to come.

Barbara Cohen 